\title{
Simulating Variable Source Problems via Post Processing of Individual Particle Tallies ${ }^{\star}$
}

\author{
D. L. Bleuel ${ }^{1,2}$, R. J. Donahue ${ }^{1}$, B. A. Ludewigt $^{1}$, and J. Vujic, ${ }^{2}$ \\ 1 Lawrence Berkeley National Laboratory, Berkeley CA, 94720, USA \\ ${ }^{2}$ University of California at Berkeley, Berkeley CA, USA
}

Topics of interest: Algorithm Developments, Variable sources, BNCT

\begin{abstract}
Monte Carlo is an extremely powerful method of simulating complex, three dimensional environments without excessive problem simplification. However, it is often time consuming to simulate models in which the source can be highly varied. Similarly difficult are optimization studies involving sources in which many input parameters are variable, such as particle energy, angle, and spatial distribution. Such studies are often approached using "brute force" methods or intelligent guesswork. One field in which these problems are often encountered is accelerator-driven Boron Neutron Capture Therapy (BNCT) for the treatment of cancers.

Solving the reverse problem of determining the best neutron source for optimal BNCT treatment can be accomplished by separating the time-consuming particletracking process of a full Monte Carlo simulation from the calculation of the source weighting factors which is typically performed at the beginning of a Monte Carlo simulation. By post-processing these weighting factors on a recorded file of individual particle tally information, the effect of changing source variables can be realized in a matter of seconds, instead of requiring hours or days for additional complete simulations. By intelligent source biasing, any number of different source distributions can be calculated quickly from a single Monte Carlo simulation. The source description can be treated as variable and the effect of changing multiple interdependent source variables on the problem's solution can be determined. Though the focus of this study is on $\mathrm{BNCT}$ applications, this procedure may be applicable to any problem that involves a variable source.
\end{abstract}

\section{Introduction to BNCT}

BNCT is a cancer treatment modality primarily aimed at glioblastoma multiforme (GBM), a non-localized malignant tumor growth in the brain. Because GBM is not confined to a single tumor mass, it is impossible to remove completely by surgery, and conventional radiation treatments and chemotherapy are highly ineffective.

To treat GBM or other cancers, BNCT utilizes a binary method. The first step involves administering a cancer-seeking pharmaceutical that has been loaded

\footnotetext{
* Supported by the U.S. Department of Energy under Contract No. DE-AC03$76 \mathrm{SF} 00098$.
} 
with ${ }^{10} \mathrm{~B}$. An ideal drug is harmless to the patient and is absorbed in cancerous cells far more selectively than in normal tissue cells. The second step is irradiation of the patient by a soft neutron beam. ${ }^{10} \mathrm{~B}$ has a very high thermal neutron capture cross section of 3830 barns and decays immediately into high LET particles via a ${ }^{10} B(n, \alpha)^{7} L i$ reaction, as well as a $480 \mathrm{keV} \gamma$ ray in $93 \%$ of the reactions. The energy of the combined high LET particles is largely confined within the diameter of a single cell, about $10 \mu \mathrm{m}$, thus targeting a large radiation dose preferentially to malignant cells without significantly irradiating healthy tissue. Because BNCT is a binary therapy, advances in both boron delivery and in neutron beam shaping are important to improving the quality of treatment.

Many different neutron sources have been proposed or used for BNCT. Two primary sources considered have been reactor sources and accelerator sources. Feasability of an accelerator-based BNCT facility has been studied at Lawrence Berkeley National Laboratory and extensively compared to several existing reactor sources[1]. The accelerator would produce neutrons via the ${ }^{7} \operatorname{Li}(p, n)^{7} B e$ reaction at proton energies at about $2.5 \mathrm{MeV}$, which produces a high neutron yield as well as a low maximum neutron energy. Epithermal neutrons in the range of about $2 \mathrm{keV}-20 \mathrm{keV}$ are most desirable[2] for BNCT. Slower neutrons do not penetrate far into the brain, where treatment is most difficult, and faster neutrons are largely absorbed in hydrogen at shallow depths.

\section{Neutron Beams}

The spectra of several different neutron beams that have been evaluated are seen in Fig. 1, demonstrating that there can be large differences in energy distribution for different beams, each of which leads to a different dose distribution in the brain. A wide variety of different spectra can be produced by various neutron sources (eg: reactors, accelerators tuned to different proton energies or utilizing different target materials) and various moderator assemblies. The clinical efficacy of each spectra must be evaluated by extensive Monte Carlo modelling with a program such as MCNP[3]. Generally, this is done in a two-step process. The first step involves modelling the neutron beam through a filter assembly[4] to determine the spectrum that will be applied to the patient, such as depicted in Fig. 1. The second step models this neutron beam emerging from the filter through an assumed patient phantom geometry to determine radiation dose distributions in the brain.

When modelling neutron beams in a head phantom, four primary reactions must be considered: Thermal neutrons produce the so-called nitrogen dose $\left(D_{N}\right)$ through the ${ }^{14} N(n, p){ }^{14} C$ reaction and the very important boron dose $\left(D_{B}\right)$. Thermal neutrons are also the main contributor to the $\gamma$ dose $\left(D_{\gamma}\right)$ via the ${ }^{1} H(n, \gamma)^{2} H$ reaction, though external $\gamma$ contamination is also important to consider. For higher neutron energies $(>1 \mathrm{keV})$, recoil protons dominate the tissue dose. The hydrogen dose $\left(D_{H}\right)$, primarily due to proton-recoil reactions, is strongly dependent on the energy spectrum of the neutron beam. Optimizing 


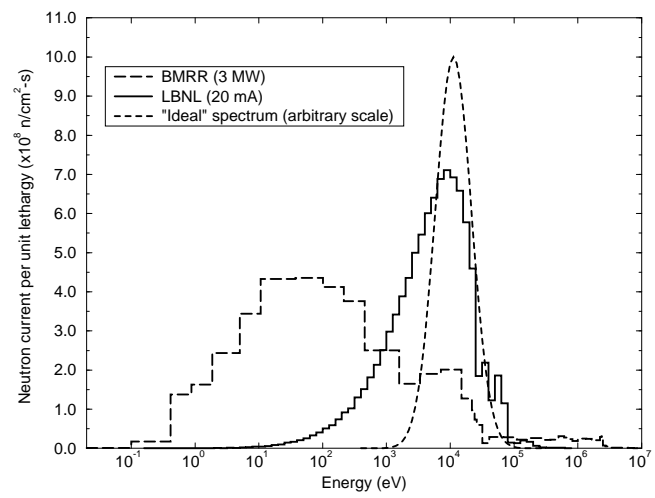

Fig. 1. Neutron energy spectra for the BMRR beam, an LBNL acclerator-based design, and an "ideal" spectrum which produces the greatest tumor dose at the brain midpoint as determined from extensive Monte Carlo optimization

neutron beams for treatment is primarily a matter of designing neutron sources, moderators, and filters to produce a spectrum which limits the unwanted "background" doses (primarily $D_{H}$, but also $D_{N}$ and $D_{\gamma}$ ) while maximizing the penetration of the beam and maintaining a high enough dose rate to treat in a reasonable amount of time.

\section{Dose Calculation}

The total effective dose to either tumor or healthy tissue is calculated by adding these different dose components, multiplied by appropriate weighting factors to obtain the total photon equivalent dose $\left(D_{t o t}\right)$ in Gray-equivalent (Gy-eq) units:

$$
D_{t o t}(x)=C F \cdot D_{B}(x)+R B E_{N} \cdot D_{N}(x)+R B E_{H} \cdot D_{r}(x)+R B E_{\gamma} \cdot D_{\gamma}(x)
$$

Doses, which vary with depth $(\mathrm{x})$ in the phantom, are multiplied by relative biological effectiveness (RBE) factors or, in the case of the boron dose, a compound factor (CF) which reflects both the boron RBE and compound specific properties. In LBNL optimization studies, the dose calculation protocol[5] developed for BNCT clinical trials at the Brookhaven Medical Research Reactor has been applied, with boron concentrations and compound factors for the boron compound boronphenylalanine (BPA) used.

These doses are calculated in small cylindrical tally volume cells $(0.4 \mathrm{~cm} \mathrm{x}$ $0.25 \mathrm{~cm}$ radius) along the neutron beam centerline in a modified Synder head phantom[2,6], after a lithiated polyethylene delimeter as shown in Fig. 2. 


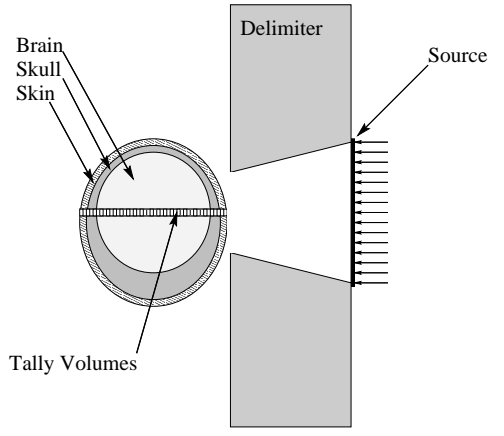

Fig. 2. Simulation geometry.

\section{The "Ubertally" Method}

Previous optimization studies have involved hundreds of Monte Carlo simulations to compare properties of different neutron beams. Some studies[2,7,8] have analyzed beam optimization on a more fundamental level, examining the effects of different energies, beam sizes, and angular distributions. While such studies offer insight into desirable beam properties, the results are often unwieldly and too specific to a particular set of treatment parameters. Small changes in phantom geometry or RBE values, for instance, can produce drastic changes in the performance of different neutron beams. Usually, the only way to determine the effect of changing one or several of the many different variables involved in a BNCT treatment is to rerun many Monte Carlo processes, each of which can take hours or days.

To accelerate the process of performing a great number of Monte Carlo simulations with the same geometry but different sources, the "Ubertally" method was devised. Instead of simulating many different sources, a single simulation is performed and source weighting factors are applied after the simulation. Several steps are involved.

\subsection{The Master Monte Carlo Run}

The first step is the MCNP input file which creates the "ubertally." In most respects, this input file is like any other MCNP input file, which a few exceptions. First, the particle source distribution is completely isotropic, uniformly distributed over a circular plane, and isoergic - a flat spectrum that covers the range of interest. For BNCT sources, the range of $0.001 \mathrm{eV}$ to $15.84893 \mathrm{MeV}$ was chosen for neutrons. To produce adequate sampling across the entire spectrum, this source is then biased by equal sampling probability in bins of equal lethargy, ten bins per decade. While the spectrum remains linearly flat for calculations, any energy bin of equal lethargy will produce the same sample frequency.

Second, the MCNP "FILES" card is used to produce two output files for the "ubertally," sequential, unformatted files named UBERTALE and UBERTALF. 
Source particle information will be stored in UBERTALE and cell tally information will be stored in UBERTALF, as described in the TALLYX subroutine.

Third, the FU card is used to call a customized TALLYX subroutine on all tally volumes.

\subsection{TALLYX}

A new tally is designed via the TALLYX subroutine in MCNP. This tally records particle information into the UBERTALE and UBERTALF files, to be processed later. These two files are obviously quite large (on order of a gigabyte), since information on every tallied particle is recorded. To save space, floating point variables are first converted to 4-byte REAL numbers and certain integers (particle type and cell number) are converted to single bytes.

When a particle track first encounters a tally volume, source information is saved into UBERTALE. The variables recorded are particle number, source position ( $\mathrm{Y}$ and $\mathrm{Z}$ ), source energy, and source angle. This information is only recorded once, even if the particle encounters several different tally volumes. Every particle track in the tally cells causes tally infomation to be saved into UBERTALF. The variables recorded in this file are particle number (for crossreferencing with the source information in UBERTALE), tally cell, particle type, tally energy, and flux (track length divided by cell volume).

\section{$4.3 \quad$ Post-processing}

With this information collected and saved, different source weights can be applied on a particle-by-particle basis, obtaining problem solutions to any number of different source distributions with the same simulation. For BNCT, doses in small cylindrical tally volumes along the beam centerline in the head phantom are calculated:

$$
D_{X}=\frac{\Psi}{N} \sum_{i=1}^{N} \Phi_{i} \cdot K_{X}\left(E_{i}\right) \cdot\left[\frac{W}{W_{o}}\right]_{i}
$$

where $X$ represents each dose component in (1), $\mathrm{N}$ is the number of particle simulations, $\Psi$ is the beam flux, $\Phi_{i}$ is the flux contribution of particle $i$ in the tally region, $K_{X}\left(E_{i}\right)$ is the energy-dependent kerma factor for each dose component, and $W$ and $W_{o}$ respectively are the new source weight and old source weight. The new source weight is the probability of the particle's energy, position, and angle in the source bin of interest. The old source weight is the probability of an equivalent bin in the original MCNP run, based on the original isoergic, isotropic, uniform distribution:

$$
W_{o}=\frac{\Delta E_{i}}{E_{\max }-E_{\min }} \cdot \frac{\Delta \mu_{i}}{\mu_{\max }-\mu_{\min }} \cdot \frac{\Delta A_{i}}{A_{t o t}}
$$

in which $\Delta E_{i}, \Delta \mu_{i}$, and $\Delta A_{i}$ are respectively the bin widths of the new source's energy, angle, and area corresponding to the original values of particle 
$i$, and the max and min values are the maximum and minimum energies of the original master Monte Carlo simulation.

These new doses are calculated and summed to produce the final result. Values of $K_{X}\left(E_{i}\right)$ can be calculated to reflect different tissue compositions as desired to determine the effect of different phantom models, though it must be understood that the material modelled for particle transport is fixed. For photon flux tallies, an effective kerma factor is calculated from mass energy absorption coefficients for average soft tissue[9]:

$$
K_{X}\left(E_{i}\right)=\frac{\mu_{e n}}{\rho} \cdot E_{i} .
$$

\section{$5 \quad$ Results}

The calculations performed in this method are exactly the same that would be performed if the source distributions were input initally into MCNP and biased by equal lethargy bins as described above. The order of the calculations has simply been altered. Instead of source weighting occuring at the beginning of each particle history, weighting is applied after the simulation has been completed. Therefore, benchmarking is straightforward. In equivalent simulations with different sources, biased as described, results are identical to the number of significant digits reported by MCNP. Fig. 3 shows the differences in the "ubertally" method compared to non-biased full MCNP simulations for several different neutron beams.

In most cases, comparison between a straight-forward MCNP simulation and the "ubertally" method is as expected. Percentage differences between the two methods is low. Since the error bars represent a single standard deviation, approximately $63 \%$ of the data points should have error bars that overlap. This appears to be the case, with the exception of one dose component. For the LBNL beam, discrepancies are apparent only in the proton-recoil dose, $D_{H}$ and only at large depths, where the dose contribution nears zero. For the BMRR beam, these proton-recoil discrepancies are more pronounced at all depths, affecting the agreement in the total dose as well. The difference in the total doses, though notable, is still low. However, this indicates that the "ubertally" method might benefit from altering the biasing of the master MCNP run to give greater emphasis to modelling higher energy particles.

These comparisons have assumed no gamma contamination is present in the neutron beam. In actual treatment planning, the gamma component is important and should be included. The post-processing "ubertally" algorithm can combine the results of a neutron and external gamma source for a true mixed beam analysis.

\section{Error Analysis}

Care must be taken when using the "ubertally" method that sources of error are considered. It is best if a much greater number of particles are simulated 

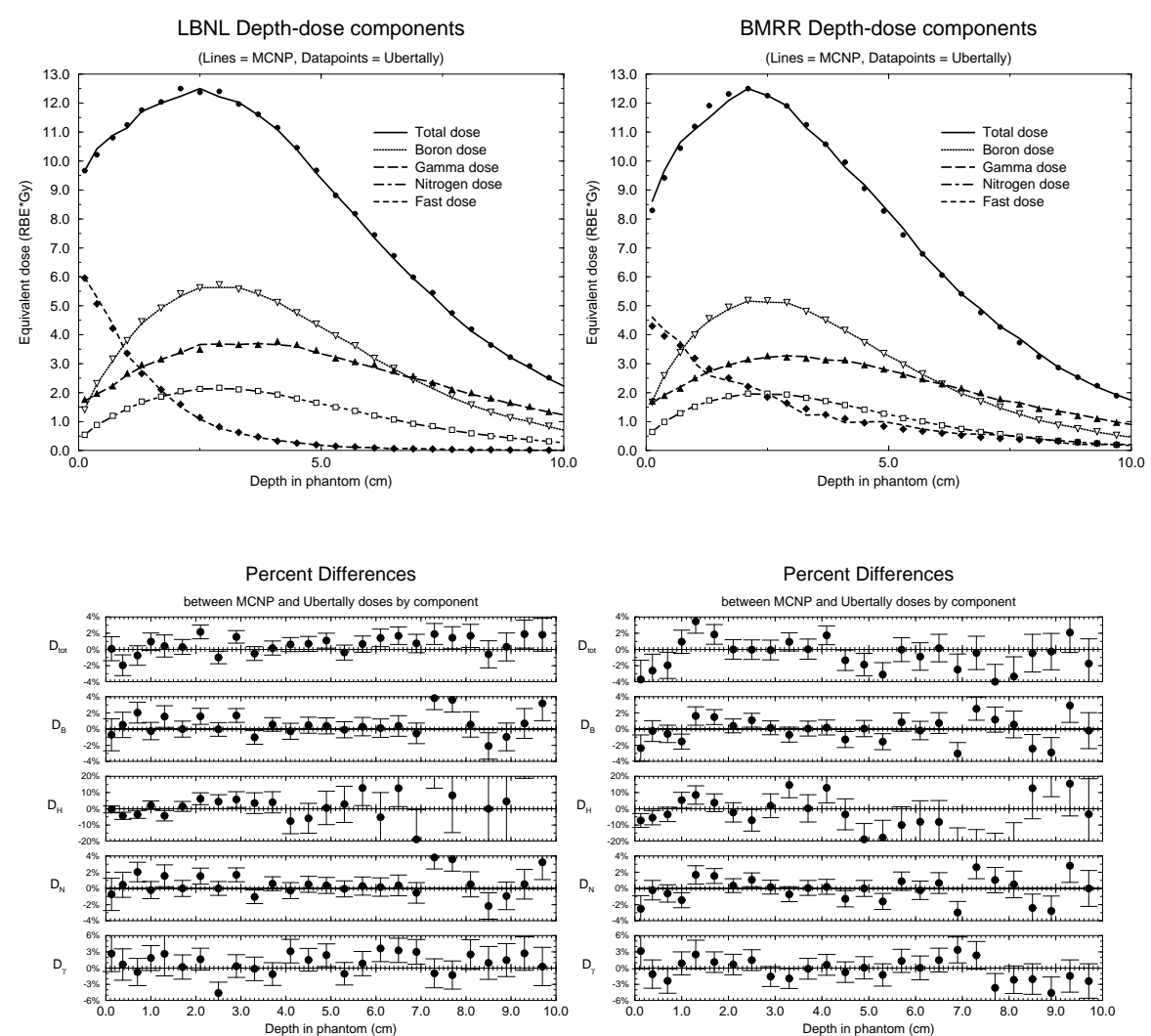

Fig. 3. Comparison of depth-dose components for healthy tissue in two different neutron beams. The data points on the top graphs represent "ubertally" values while the lines represent values of a normal MCNP run. The bottom graphs show the percentage variations of these values, with error bars representing one standard deviation statistical error, added in quadrature from each simulation.

in the master run than are normally required, as many simulated particles may be wasted in the final calculation, especially if the source applied is narrow in energy, angle, and/or spatial distribution. Fifty million particles were sufficient to produce low statistical errors, though additional particles, especially biased to higher energies, may be needed.

It should also be noted that because a single Monte Carlo simulation can be applied to many different sources, any source of error in the original run will be applied to all results. Thus, comparison between "ubertally" results can be misleading and the ten statistical checks MCNP normally make are not necessarily applicable. Only the relative error is calculated by the "ubertally." 


\section{Conclusions}

The "ubertally" method of applying source weighting factors to readjust a Monte Carlo simulation for different variable sources is quite powerful. It can eliminate the need for many lengthy simulations when the source can change, but the environment geometry does not. A simulation that normally takes up to a full day of computation can be accomplished in 10-15 minutes. More error analysis studies are needed to ensure that results are accurate and not misleading. Future study will utlilize the "ubertally" to produce high-resolution response functions of dose vs. neutron energy, angle, beam size, phantom size, and RBEs.

\section{References}

1. Bleuel, D. L., Donahue, R. J., Ludewigt, B. A., and Vujic, J., "Designing Accelerator-based Epithermal Neutron Beams for Boron Neutron Capture Therapy," Medical Physics 25, 9, pp. 1725-1734 (1998).

2. Bleuel, D. L., Donahue, R. J., Ludewigt, B. A., and Vujic, J., "Development of a Neutron Energy-Biased In-Air Figure of Merit for Predicting In-Phantom BNCT Neutron Beam Characteristics," presented at the Eighth International Symposium on Neutron Capture Therapy for Cancer in La Jolla, CA, Sep. 13-18, 1998.

3. Briesmeister, J. F., MCNP - A General Monte Carlo N-Particle Transport Code, Version 4B, Los Alamos National Lab, 1997.

4. Bleuel, D. L., Chu, W. T. Donahue, R. J., Ludewigt, B. A., McDonald, R. J. Smith, A. R., Stone, N. A., Vujic, J. "Initial Experimental Verification of the Neutron Beam Modeling for the LBNL BNCT Facility," Applications of Accelerators in Research and Industry, Proceedings of the Fifteenth International Conference, November 4-7, 1998, Denton, Texas, AIP Conference Proceedings 475 Vol. 2, pp. 1050-1055 (1999)

5. Coderre, J. A., Bergland, R., Capala, J., Chadha, M., Chanana, A. J., Elowitz, E., Joel, D. D., Liu, H. B., Slatkin, D., "Boron Neutron Capture Therapy for Glioblastoma Multiforme using $p$-boronophenylalanine and Epithermal NeutronsTrials Design and Early Clinical Results,” J. Neuro. Oncol. 33, pp. 93-104 (1997).

6. Snyder, W. S., Fisher, Jr., H. L., Ford, M. R., Warner, G. G., "Estimates for Absorbed Fractions for Monoenergetic Photon Sources Uniformly Distributed in Various Organs of a Heterogeneous Phantom (Appendix B)," J. Nucl. Medicine, MIRD Supplement No. 3, Pamphlet 5 (1969).

7. Yanch, J. C., Harling, O. K., "Dosimetric Effects of Beam Size and Collimation of Epithermal Neutrons for Boron Neutron Capture Therapy," Radiation Research 135, pp. 131-145 (1993).

8. Verbeke, J. M., Vujic, J., and Leung, K. N. "Neutron Beam Optimization for BNCT Using the DD and DT High Energy Neutron Sources," Nuclear Technology 129, 2, pp. 257-278, (2000)

9. "Photon, Electron, Proton and Neutron Interaction Data for Body Tissues," ICRU Report 46, February 28, (1992). 